\title{
Ameloblastic Fibro-Odontoma
}

\author{
Brenda L. Nelson $\cdot$ Lester D. R. Thompson
}

Received: 26 September 2013/Accepted: 18 October 2013/Published online: 7 November 2013

(C) Springer Science+Business Media New York (outside the USA) 2013

\begin{abstract}
A case of an amelobastic fibro-odontoma affecting a 15 year-old girl will be discussed. The characteristic radiologic and histologic features of the entity will be described.
\end{abstract}

Keywords Amelobastic fibro-odotoma - Maxilla . Impacted teeth · Radio-opacities · Odontogenic epithelium $\cdot$ Stroma

\section{History}

A 15-year old female presented to her dentist for a routine appointment. A swelling of the left anterior maxilla was noted during clinical examination, which the patient recalled noticing for the past 5 to 6 months. She denied pain and there was no history of trauma or infection. Intraoral examination revealed the absence of the left maxillary first and second molars.

Disclaimer: The opinions and assertions expressed herein are those of the author and are not to be construed as official or representing the views of the Department of the Navy or the Department of Defense or Southern California Permanente Medical Group.

B. L. Nelson $(\square)$

Department of Anatomic Pathology, Naval Medical Center San Diego, 34800 Bob Wilson Drive, San Diego, CA 92134-5000, USA

e-mail: brenda.nelson@med.navy.mil

L. D. R. Thompson

Department of Pathology, Southern California Permanente

Medical Group, Woodland Hills Medical Center, 5601 De Soto

Avenue, Woodland Hills, CA 91365, USA

e-mail: Lester.D.Thompson@kp.org

\section{Radiographic Features}

Computed tomography (CT) of the head revealed a welldefined unilocular radiolucent lesion of the anterior maxilla measuring $2.9 \mathrm{~cm}$ in greatest dimension. The lesion was notable for radio-opacities that ranged from delicate and flake-like to larger dense aggregations (Fig. 1). The first and second molar were impacted and intimately associated with the lesion (Fig. 2).

\section{Diagnosis}

Histological examination of hematoxylin and eosin stained slides revealed a biphasic tumor which consisted of narrow islands and cords of odontogenic epithelium in a myxoid cellular stroma. The epithelial islands show peripheral columnar cells surrounding a looser collection of spindle cells while the stroma is cellular, composed of stellate and spindle shaped cells (Fig. 3). Throughout the lesion the products of odontogenesis were readily identified: mature tubular dentin and enamel matrix (Fig. 4).

\section{Discussion}

Ameloblastic fibro-odontoma (AFO) is defined by the World Health Organization (WHO) as a neoplasm consisting of odontogenic ectomesenchyme resembling the dental papilla, epithelial strands and nests resembling dental lamina and enamel organ in conjunction with the presence of dentin and enamel [1]. AFO is a mixed odontogenic tumor and shares features with ameloblastic fibroma (AF) and odontoma. Some have suggested that these lesions are in fact one entity identified at different 


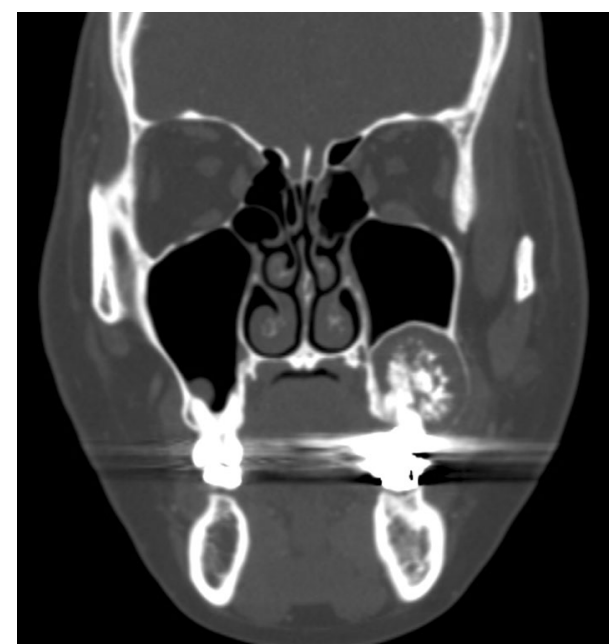

Fig. 1 Computerized tomography (CT) shows a radiolucent defect of the left maxilla with small to medium sized areas of calcification

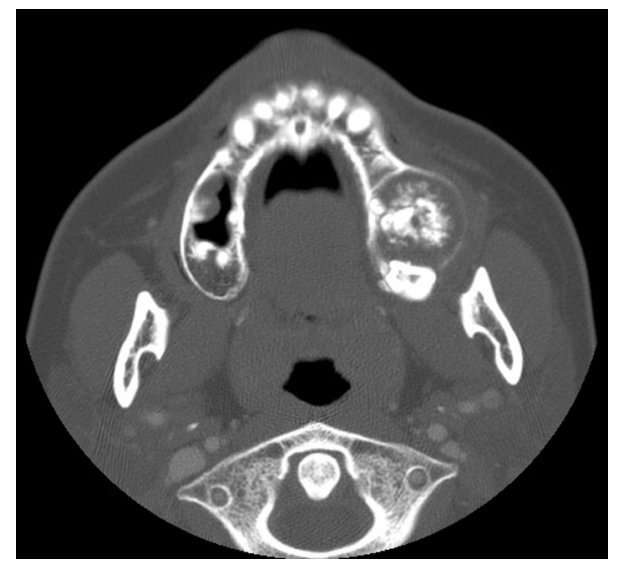

Fig. 2 The axial view shows the same characteristics with the impacted molar identified in the posterior part of the tumor

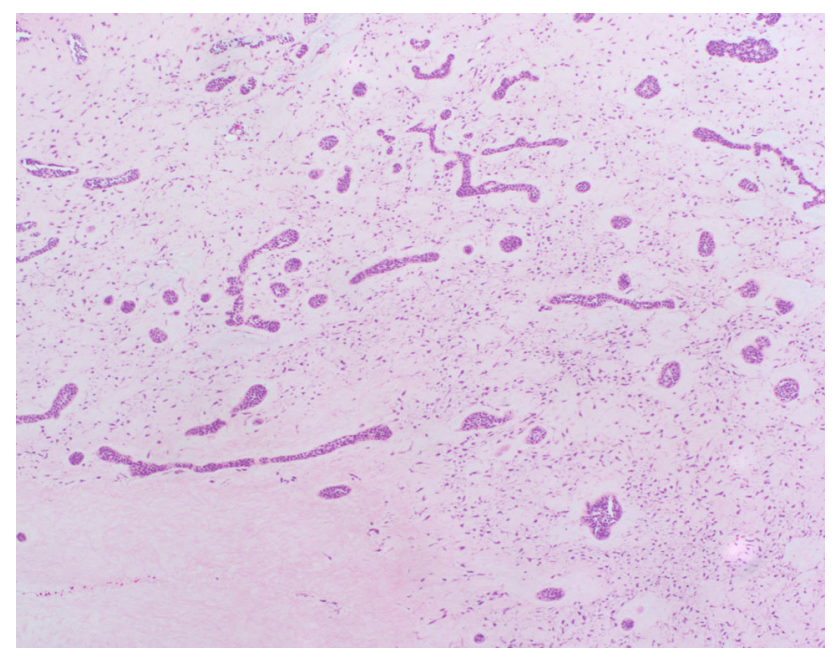

Fig. 3 Long, narrow cords of odontogenic epithelium in a cellular stroma

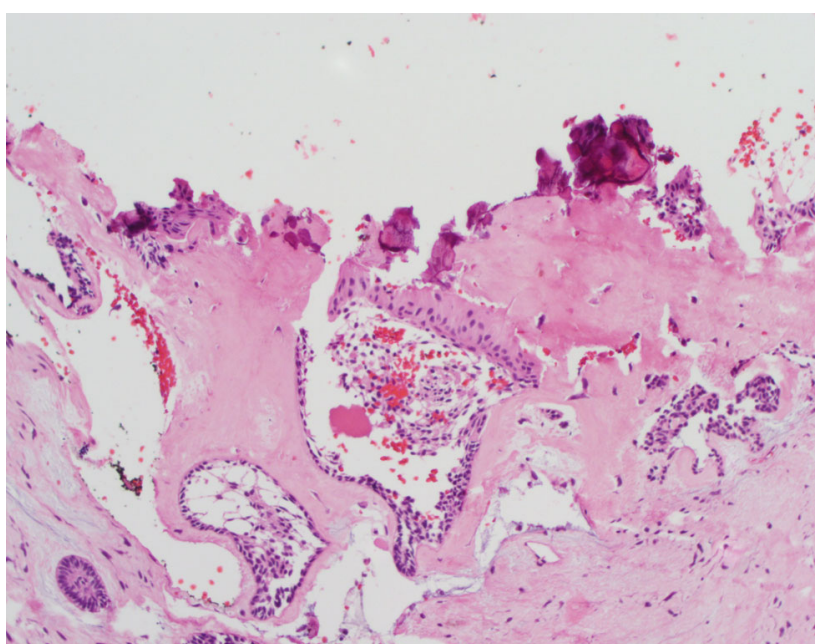

Fig. 4 The soft tissue component of the tumor intermixed with the calcified products of odontogenesis

stages of development: an ameloblastic fibroma becomes an AFO as it matures and in turn, it may develop into an odontoma. However, as AFO presents, on average, in a younger age group than a patient with $\mathrm{AF}$, this suggestion can be refuted. Despite histologic similarities these tumors should be considered different and distinct entities.

The AFO is a rare benign tumor representing approximately $3 \%$ of all odontogenic tumors. It usually occurs in the first two decades of life, with an average age of 9 years [2]. No gender predilection has been identified [3]. Patients commonly present with a painless, slow growing swelling and/or failure of tooth eruption [3-5]. There is no predilection for the mandible or maxilla, although it tends to favor the posterior areas.

Imaging generally shows a well-defined radiolucency with variable amounts of randomly distributed radioopaque material. Ameloblastic fibro-odontomas are frequently associated with unerupted teeth, occasionally causing dramatic displacement [5]. Smaller lesions may be incidental findings on routine dental radiographs.

Histologically, AFOs are composed of islands, cords and strands of distinctive odontogenic epithelium in a cellular stroma, its components resembling stages of the developing tooth. The epithelium is characterized by peripheral palisading of columnar cells that surround loose spindled epithelium, resembling stellate reticulum. The epithelial component shares many features of ameloblastoma however, the stroma is strikingly different. The stroma is a cellular ectomesenchyme made up of spindle-shaped cells that resemble the dental papilla. The presence of dentin and enamel matrix (mature enamel is lost during processing) is the feature that separates the AFO from ameloblastic fibroma. The amount of mineralized products of odontogenesis may vary, from being easily seen on grossing to requiring serial sectioning to identify dentin and enamel matrix microscopically. 
The treatment of AFO is enucleation or curettage since it is considered a non-aggressive lesion. AFO are reported to recur only rarely [5]. Unlike ameloblastic fibroma, AFO is not generally associated with a malignant transformation to ameloblastic fibrosarcoma [6]. This difference in malignant potential further supports the separation of these two tumors.

\section{References}

1. Barnes L, Eveson JW, Reichart P, Sidransky D, eds. WHO international histological classification of tumors, Volume 9. IARC Press; 2005. p 308-309.

2. Slootweg PJ. An analysis of the interrelationship of the mixed odontogenic tumors-ameloblastic fibroma, ameloblastic fibro- odontoma, and the odontomas. Oral Surg Oral Med Oral Pathol. 1981;51(3):266-76.

3. Oghli AA, Scuto I, Ziegler C, Flechtenmacher C, Hofele C. A large ameloblastic fibro-odontoma of the right mandible. Med Oral Patol Oral Cir Bucal. 2007;12(1):E34-7.

4. Pontes HA, Pontes FS, Lameira AG, Salim RA, Carvalho PL, Guimarães DM, Pinto DD Jr. Report of four cases of ameloblastic fibro-odontoma in mandible and discussion of the literature about the treatment. J Craniomaxillofac Surg. 2012;40(2):e59-63.

5. De Riu G, Meloni SM, Contini M, Tullio A. Ameloblastic fibroodontoma. Case report and review of the literature. J Craniomaxillofac Surg. 2010;38(2):141-4.

6. Müller S, Parker DC, Kapadia SB, Budnick SD, Barnes EL. Ameloblasticfibrosarcoma of the jaws. A clinicopathologic and DNA analysis of five cases and review of the literature with discussion of its relationship to ameloblastic fibroma. Oral Surg Oral Med Oral Pathol Oral Radiol Endod. 1995;79(4):469-77. 